\title{
Referentes conceptuales para el abordaje de las representaciones sociales de la paz
}

\section{Conceptual referents for the approach of the social representations of peace}

\section{Referenciais conceituais para a abordagem das representações sociais da paz}

\author{
M. DH. Evelyn Cerdas Agüero \\ Doctoranda en Educación \\ Máster en Derechos Humanos y Educación para la Paz \\ Académica en el Instituto de Estudios Latinoamericanos \\ Universidad Nacional, Costa Rica. \\ Recibido: 10/05/2018 Aceptado: 30/05/2018
}

\section{Resumen}

Este trabajo aborda de forma conceptual las representaciones sociales y su relación con los aspectos de la paz positiva y la paz negativa. El objetivo de este ensayo se centra en abordar elementos teóricos acerca de los conceptos de paz negativa y paz positiva que inciden en las representaciones sociales (RS). Para esto se hizo una investigación de los conceptos y se procedió a realizar un análisis de contenido de estos para su reflexión. Se concluye que las reflexiones y análisis de elementos teóricos acerca de los conceptos de paz negativa y paz positiva permiten

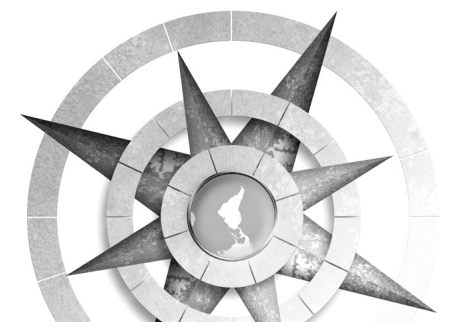

DOI: https://doi.org/10.15359/tdna.34-64.7 reconocer que muchos de los significados de paz que surgen de la realidad se construyen por influencia de conceptos que rigen los sistemas sociales, la cultura y las instituciones y, en gran manera, las representaciones sociales acerca de la paz son conformadas por la influencia de informaciones mediatizadas por una intencionalidad.

Palabras clave: paz, paz positiva, paz negativa, representaciones sociales.

\section{Abstract}

This paper deals conceptually with social representations and their relationship to aspects of positive peace and negative peace. The purpose of this essay is to address theoretical elements about the concepts of negative peace and positive peace that affect social representations (SR). For this purpose, the concepts were researched and an analysis of their content was carried out for reflection. It is 
concluded that the reflections and analysis of theoretical elements about the concepts of negative peace and positive peace allow us to recognize that many of the meanings of peace that arise from reality are constructed by the influence of concepts that govern social systems, culture and institutions and, to a great extent, social representations about peace are shaped by the influence of information mediated by an intention.

Keywords: Social Representations (SR), peace, positive, peace, negative peace, Pease Studies in Latin America.

\section{Resumo}

Neste ensaio lida conceitualmente com representações sociais e sua relação com aspectos de paz positiva e paz negativa. O objetivo deste ensaio é abordar elementos teóricos sobre os conceitos de paz negativa e paz positiva que afetam as representações sociais (RS). Para tanto, os conceitos foram pesquisados e uma análise de seu conteúdo foi realizada para reflexão. Conclui-se que as reflexões e análises de elementos teóricos sobre os conceitos de paz negativa e paz positiva nos permitem reconhecer que muitos dos significados da paz que emergem da realidade são construídos pela influência de conceitos que regem os sistemas sociais, a cultura e as instituições. e, em grande medida, as representações sociais sobre a paz são moldadas pela influência da informação mediada por uma intenção.
Palavras chave: representações sociais, paz, paz negativa, paz positiva, Estudos de paz na América Latina.

\section{Introducción}

Este artículo tiene como objetivo presentar elementos teóricos acerca de los conceptos de paz negativa y paz positiva que inciden en las representaciones sociales (RS) de la paz. Para ello se procedió a realizar una búsqueda bibliográfica para describir los conceptos de paz y hacer un análisis de contenido para establecer su relación con las representaciones sociales.

La inquietud de escribir este artículo de investigación surge de considerar que uno de los elementos que constituyen las representaciones sociales es la información, la cual se manifiesta y transmite, además de otras formas, por medio de las conceptualizaciones elaboradas respecto de un objeto dado, en este caso la paz.

Las representaciones sociales son una forma de interpretación de la realidad social cotidiana, interpretación que tiene influencia de lo que el ser humano conoce y aprende desde su realidad social, cultural y educativa. También, en este aspecto la educación es un factor importante porque transmite, analiza y critica

110 Referentes conceptuales para el abordaje de las representaciones sociales de la paz Evelyn Cerdas Agüero

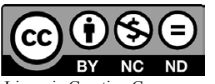

Licencia Creative Commons Atribución-No-Comercia SinDerivadas 3.0 Costa Rica

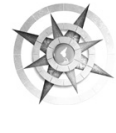


conceptos y abordajes teóricos que conforman gran parte de las creencias, actitudes, informaciones y opiniones que conforman las representaciones acerca de la paz. De ahí se parte para entender que las personas pueden explicar y analizar la paz por medio de los conceptos o los abordajes conceptuales que han asumido y aprendido por medio de la información que han recibido, pues estos forman parte de su cognición.

La relación entre la paz y las representaciones sociales es que ambas se definen en un medio social configurado, entre otros aspectos, por informaciones y diversas concepciones de mundo, se centran en los seres humanos, los sujetos y reciben influencia de aspectos de la cultura, la educación, las experiencias y los valores.

En lo que concierne a este trabajo se analizan los aspectos conceptuales de la paz como una representación configurada por informaciones, cuya base es, por lo tanto, los componentes del concepto de paz positiva y negativa. Los conceptos constituyen parte del conocimiento acumulado que justifica la realidad y lo que las personas piensan, además, generan los lineamientos que justifican la forma de actuar de las personas (en concordancia con estos); también inciden en las actitudes, creencias y opiniones que constituyen la representación y viceversa, pero que no son abarcadores de toda la representación social, sino parte constitutiva de la información de esta.

El artículo se estructura, inicialmente, a partir de realizar una conceptualización de las representaciones sociales, su contenido, funciones y su relación con las acciones de las personas para, seguidamente, abordar los conceptos de paz negativa y de paz positiva.

\section{Las Representaciones Sociales}

De acuerdo con los planteamientos de Moscovici (2008) las representaciones sociales circulan a diario y se revelan por medio de palabras, gestos y encuentros, se manifiestan en las relaciones, los objetos y la comunicación, corresponden a dos aspectos: el simbólico, por el cual son elaboradas, y la práctica que las produce. Existen siempre que haya un objeto y un sujeto, sin embargo, esto no implica que se vean de forma separada, pero sí es condición para su existencia, de acuerdo con Jodelet (2007), la representación es de un objeto o de un sujeto.

Se refieren a las diversas formas de pensar de las personas, de forma individual y como parte de un

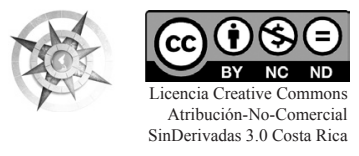

Referentes conceptuales para el abordaje de las representaciones 111

sociales de la paz

Evelyn Cerdas Agüero 
determinado grupo social, esto es, de acuerdo con Banchs (2001), que el estudio de las representaciones sociales busca conocer las formas de pensar de las personas, así como las formas en las que como parte de una colectividad construyen la realidad.

Esta construcción, también se da desde el conocimiento teórico, mediado, en gran manera por el espacio educativo en el cual el sujeto aprende una serie de conceptos que perfilan ese bagaje cognoscitivo; así como por el contexto sociocultural y las experiencias de vida. Los diversos conceptos que se han formulado y prevalecen en la historia y en los contextos educativos influyen como información en las representaciones sociales que tienen las personas y los grupos acerca de la paz, pero a la vez constituyen representaciones en el nivel cognoscitivo del concepto de paz que permean el discurso educativo, social, cultural y político.

Así, existen una serie de elementos participantes y condicionantes en la construcción de una representación, como por ejemplo, los conocimientos que se logran por medio de las experiencias personales, el contexto familiar y comunitario, hasta el sistema educativo y los valores presentes en la cultura. Esto significa, según Moscovici (1979, p. 43) que la representación "(...) es una forma de conocimiento a través de la cual el que conoce se coloca dentro de lo que conoce". Es decir, la persona se ubica en su espacio cotidiano, su realidad y sus experiencias, lo que conlleva a que haya una constante entre representar y representarse, es una organización significante que da sentido a la realidad, no solo se representa a sí misma como sujeto que participa y construye la realidad, sino que representa lo que esta significa. Es una forma de conocimiento, de acuerdo con Jodelet (2007), concebido como el conocimiento ordinario, el sentido común, construido en la sociedad.

Entonces se puede decir que las representaciones sociales se refieren al conocimiento basado en las experiencias cotidianas, los discursos, el lenguaje, los aprendizajes, las informaciones (elementos socioculturales) en el nivel personal y grupal, que permiten leer, interpretar y dar significado a la realidad, acontecimientos o fenómenos de esta, incluidas las relaciones sociales, como si fueran los anteojos por medio de los cuales se observa y da significado. En este sentido, de acuerdo con Ibáñez (2001) estas se comprenden porque hay un sujeto que pertenece a un determinado grupo social y asigna significados al objeto de la realidad, los cuales se basan en las interacciones sociales de este.

112 Referentes conceptuales para el abordaje de las representaciones sociales de la paz Evelyn Cerdas Agüero

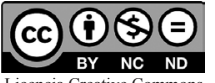

Licencia Creative Commons Atribución-No-Comercia SinDerivadas 3.0 Costa Rica

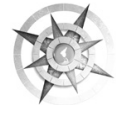




\section{Contenido de las representacio- nes sociales}

Las representaciones sociales están constituidas por un conjunto de informaciones, creencias, opiniones y actitudes con respecto a un determinado objeto (Abric, 2001; Moscovici, 1979). En relación con las informaciones, que es el tema que atañe, estas se basan en conceptos y contenidos, experiencias, ideologías, aprendizajes desde la educación no solo formal, sino informal y no formal. Además, generan los datos y las explicaciones que tienen las personas acerca de la realidad (Araya, 2002).

De acuerdo con Araya (2002, p. 40) la información se refiere a "la organización de los conocimientos que tiene una persona o grupo sobre un objeto o situación social determinada". Con respecto a esta se deben considerar los siguientes elementos: la cantidad, la calidad, la precisión, el origen (contacto directo con el objeto o por medio de la comunicación social), su carácter (estereotipado o no) y la pertenencia grupal del sujeto. Para Moscovici (1979, p. $45)$, "se relaciona con la organización de los conocimientos que posee un grupo con respecto a un objeto social". Así, se trata de lograr conocer cómo los aspectos conceptuales que dan fundamento a la paz se formulan como informaciones que se transmiten y explican desde diversos ámbitos comunicativos (cultura, educación, familia, lecturas, medios de comunicación, etc.).

Es importante reconocer el papel de la comunicación social, cuando esta da origen a las informaciones desde los medios de comunicación, la religión, la cultura, entre otros, e influyen de manera importante en las informaciones que recibe cada sujeto y que, a la vez, son parte constitutiva de las informaciones. Esta dimensión conduce, necesariamente, a la riqueza de datos o explicaciones que sobre la realidad se forman las personas en sus relaciones cotidianas.

Sin embargo, hay que considerar que las pertenencias grupales y las ubicaciones sociales mediatizan la cantidad y la precisión de la información disponible. Por ejemplo, se considera importante mencionar que las informaciones que provienen de las instituciones como las educativas formales están mediatizadas por determinados intereses ideológicos y políticos que tienen diversas intenciones, considerando que la educación tiene una intencionalidad, la cual se ve reflejada en las informaciones estructuradas y abordajes teóricos que se incorporan en los planes de estudio para explicar la

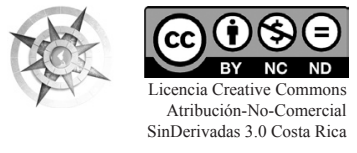

Referentes conceptuales para el abordaje de las representaciones 113

sociales de la paz

Evelyn Cerdas Agüero 
realidad desde diversas perspectivas y generar aprendizajes.

Efectivamente, el sistema educativo tiene una intencionalidad con los conceptos de paz por medio de los cuales quiere incidir en la formación de los ciudadanos y las ciudadanas para que actúen en consonancia con estos. De esta forma, según Materán (2008) las representaciones sociales se vinculan a las prácticas sociales y culturales lo que les impregna de uno de sus aspectos fundamentales, estas van más allá de lo subjetivo, y permean la cultura y la sociedad.

\section{Funciones de las representacio- nes sociales}

Las representaciones sociales desempeñan funciones importantes en la sociedad, en los grupos y en las prácticas sociales que permiten comprender la realidad, las dinámicas que se dan en la sociedad y entre las personas, esto es, de acuerdo con Valencia (2007, p. 51) que dan "sentido a la construcción de una realidad cotidiana, compartida y estructurada por los grupos, en el seno de los cuales son elaboradas". Jodelet (2007) también plantea que estas codifican y clasifican la realidad, de forma que se logra tener criterios para construir los significados que están presentes y son compartidos en la sociedad.
Es así como hay un vínculo entre la experiencia personal, lo grupal y lo social para dar sentido a la realidad.

De acuerdo con Abric (2001) las representaciones sociales cumplen funciones de saber, identidad, orientación y justificación.

Funciones de saber: son funciones cognitivas por medio de las cuales las personas o grupos comprenden y explican la realidad, esto a través del sentido común como forma de conocimiento. Están vinculadas con la comunicación como una forma para intercambiar los saberes del sentido común. Esta función de saber se refiere, también, a las explicaciones de la realidad y de las conductas de las personas basadas en los referentes conceptuales, desde el punto de vista cognitivo. Así se reconoce que la aprehensión de determinados conceptos genera una serie de conocimientos teóricos que funcionan como herramientas para analizar y explicar la realidad social en torno a lo que se concibe o no como paz, sus características y manifestaciones.

Funciones identitarias: permiten que las representaciones mantengan y definan la identidad de las personas y los grupos sociales. Es importante mencionar que las conceptualizaciones y las informaciones son

114 Referentes conceptuales para el abordaje de las representaciones sociales de la paz Evelyn Cerdas Agüero

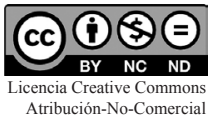


un insumo que ayuda a conformar la identidad como una forma de conocimiento que sustenta las creencias, las acciones, la forma de resolver conflictos y el sentido de pertenencia a un grupo determinado.

Funciones de orientación: por medio de las cuales las representaciones orientan las conductas de las personas en los grupos y en la sociedad. De acuerdo con Abric (2001, p. 16), "La representación produce igualmente un sistema de anticipaciones y expectativas. Es así, pues, una acción sobre la realidad: selección y filtro de las informaciones, interpretaciones con objeto de volver a esa realidad conforme a la representación". Esta acción sobre la realidad estaría, en gran manera, basada en determinadas concepciones que rigen la forma de actuar de las personas.

Los referentes conceptuales de la paz orientan, sin limitarse solo a esto, el análisis de las conductas presentes en la sociedad, de forma que se categorizan aquellas conductas que buscan, promueven, construyen la paz y aquellas que no, con base en las definiciones que se tienen de esta, sus características y sus principios. Las concepciones, también, plantean los principios que deben guiar la forma en la que las personas actúan, pues dan algunos parámetros a seguir. Es así como el tener un concepto de paz determinado puede prescribir en gran manera las conductas para que coincidan con este.

En este aspecto, los componentes conceptuales modelan las diversas formas de comportamiento y las características que debería cumplir el medio social para construir, promover y consolidar la paz.

Funciones de justificación: buscan dar una justificación a los comportamientos y las acciones realizadas, para que sean aceptados, normalizados o perpetuados. En lo que se refiere a esta función, las nociones de paz justifican las acciones sociales y políticas para su mantenimiento, construcción y promoción, las conductas socialmente aceptables y las acciones educativas que la promueven.

De tal forma que se considera que los conceptos que se han aprehendido funcionan para justificar los resultados que se esperan de la paz y los prerrequisitos para que esta se dé. Las concepciones que se tienen son parte de la formación de la representación social, considerando que existe una relación estrecha entre la información conceptual que se maneja y la construcción de la representación social, además, inciden en las acciones que podrían ser acordes o no con

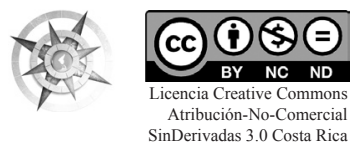

Referentes conceptuales para el abordaje de las representaciones 115

sociales de la paz

Evelyn Cerdas Agüero 
los planteamientos y las justifican. Estas concepciones forman parte de lo que es aceptable o no en términos de normas y conductas sociales.

La representación "funciona como un sistema de interpretación de la realidad que rige las relaciones de los individuos con su entorno físico y social, ya que determinará sus comportamientos o sus prácticas" (Abric, 2001, p. 13). En este sentido, las representaciones que se forman a partir de determinados componentes conceptuales permiten interpretar y explicar la realidad con la ayuda de los "lentes" de estos conceptos, situar las relaciones, las interacciones individuales y colectivas en el marco de lo que es aceptable o no para que haya paz, como respuesta a lo que se concibe.

No se puede obviar que estas concepciones están inmersas en justificar lo que la sociedad desea y orienta, por medio del aprendizaje, las acciones que se llevan a cabo en la sociedad y que se espera que sean acordes con las normas sociales que promueven la paz. La situación empieza a modelarse cuando los conceptos se estiran de manera que se asume que la paz no se resume en normas sociales, sino que se amplía a acciones, actitudes, formas de convivencia, derechos humanos y oportunidades.
Estas funciones de las representaciones permiten ver la relación existente entre las representaciones y las prácticas sociales, por lo cual los conceptos que se tienen, los aprendizajes, los valores, las creencias influyen en estas acciones, las justifican, las orientan y son bases para determinar la forma de ver la realidad. Además, permiten ver como el sujeto organiza su concepción de mundo con base en lo que considera como paz dado el acervo conceptual que posee.

\section{El contexto y su vínculo con las representaciones sociales}

Resulta importante mencionar que las representaciones sociales, son también determinadas por el contexto. De acuerdo con Cuevas y Mireles (2016) el contexto rodea a los sujetos, son las situaciones que viven, las jerarquías sociales o institucionales establecidas, los aspectos culturales, los valores y las normas.

Según Ibáñez (2001) las representaciones son elaboradas en un contexto histórico y social, lo cual quiere decir que quien las elabora pertenece o se desarrolla en un espacio social, cultural e histórico que a la vez reflejan este. Abric (2001) habla de un contexto discursivo y uno social. El discursivo se refiere a las condiciones en las que se produce el discurso, es

116 Referentes conceptuales para el abordaje de las representaciones sociales de la paz Evelyn Cerdas Agüero 
a partir de este que se formula una representación, se relaciona con el público al que se dirige y el grupo o personas desde el cual proviene. El contexto social hace referencia al espacio que ocupa el individuo o grupo en la sociedad, así como a las relaciones sociales en las que interactúa. Este planteamiento también lo trae a relucir Moscovici (1979), el cual menciona que al hablar de representaciones sociales hay un vínculo entre el individuo y su contexto, porque el individuo o grupo no se aísla de su contexto social, cultural, político, económico e histórico.

Por otro lado, Jodelet (2007) alude a tres contextos (o esferas de pertenencia de las representaciones sociales): el subjetivo, intersubjetivo y trans-subjetivo. El subjetivo explica que el sujeto es parte de un espacio social, es participante y constructor en un contexto social determinado, de forma que elabora las representaciones basándose en sus experiencias individuales (emociones, afectividad, historia, conocimientos). Son "procesos a través de los cuales el sujeto se apropia y construye esas representaciones (...)” (p. 206). Esto implica la visión de un sujeto que se sitúa en el contexto social, con identidad y con características emocionales propias y que a la vez interviene en la construcción de representaciones o la adhesión a las que han sido construidas por otros.

Además, se reconoce que el sujeto no está aislado de su realidad, sino que forma parte de un contexto específico que lo educa, lo predispone, lo condiciona y hasta lo somete, las experiencias y los resultados de estos procesos marcan la construcción de las representaciones sociales de forma individual.

El contexto intersubjetivo se refiere al que se genera entre las personas, en el cual las interacciones entre individuos o grupos se constituyen como la base para la construcción de representaciones sociales, por medio de la comunicación directa (opiniones, conocimientos, visiones de la realidad y formas de pensamiento). Este contexto infiere el sentido de comunidad y de pertenencia a un determinado grupo social en el cual se generan interacciones entre los sujetos, las cuales les permiten tener experiencias que trascienden el ámbito individual, por medio de intercambios con otros actores sociales que pueden tener una influencia significativa en la formación o elaboración de representaciones sociales del individuo.

El contexto trans-subjetivo se refiere al espacio público y social en el cual circulan las representaciones

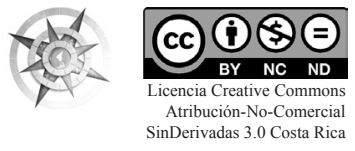

Referentes conceptuales para el abordaje de las representaciones 117 sociales de la paz Evelyn Cerdas Agüero 
sociales, está constituido por elementos intersubjetivos y subjetivos, desde el nivel micro hasta el macro, incluye los individuos, los contextos de interacción y los sistemas o instituciones.

Esto quiere decir que en estos espacios las representaciones sociales provienen de un sinnúmero de fuentes como los medios de comunicación, las ideologías, la cultura, las instituciones, las relaciones de poder, los sistemas sociales, las normas y los valores. Además, implica no solo las formas de pensar, sino las acciones, las experiencias de vida, los conocimientos y las emociones de cada individuo.

\section{Presupuestos acerca de la paz}

La paz abarca una concepción compleja, ha sido concebida como un valor humano, como una praxis social y como un derecho humano, además, se habla de diferentes concepciones de paz en las culturas, desde la paz personal hasta la paz social. En este aspecto se hará un abordaje teórico acerca de las ideas de paz, en torno a los conceptos de paz negativa y paz positiva. Las diversas concepciones de paz obedecen a una serie de aspectos sociales, políticos y culturales que estructuran las representaciones sociales que tienen los individuos y los grupos de esta, dependiendo del contexto, la edad y el aprendizaje.

En el siguiente apartado se abordarán dos tipologías de la paz: la positiva y la negativa, planteadas por Galtung (2003) .

\section{La concepción negativa de la paz}

La paz negativa se ha considerado como el primer concepto que surgió para estudiar la paz (Jiménez, 2009; Bobbio, 1982), se refiere a la falta de conflictos armados y la violencia expresa (Jiménez, 2009), a la ausencia de la violencia directa o cualquier tipo de violencia (Galtung, 2003). Este concepto se origina en la antigua Roma como absentia belli o ausencia de guerra, una paz que estaba garantizada por el poder militar del imperio. A este se le aúna el concepto de pax romana, en el cual se considera la paz como la ausencia de guerra o cualquier conflicto bélico, esto ha conducido a considerar que la paz depende del papel de los Estados para su realización. "La pax romana constituía todo un sistema de orden, control y relación legal, era ausencia de violencia, pero no garantizaba justicia y prosperidad" (Jiménez, 2009, p. 147). Así, esta concepción plantea la necesidad de defenderse de forma militar ante los enemigos, lo cual incluye el poder militar y el

118 Referentes conceptuales para el abordaje de las representaciones sociales de la paz Evelyn Cerdas Agüero 
armamentismo, la utilización del poder para defenderse y para amenazar, así la paz estaría asegurada para el más fuerte y con más poder militar. Según Jares (1999), en este sentido la paz se logra si existe unidad al interior de los Estados y la defensa frente a amenazas externas.

Las representaciones sociales que giran en torno a los planteamientos de paz negativa generan una visión de ausencia de guerra, conflictos armados, guerrillas y terrorismo; es una visión basada en la historia y en el sentido colectivo que se circunscribe en un nivel macro, de Estado, naciones y relaciones políticas y diplomáticas. Así, el Estado debe asegurar la paz interna por medio de sus instituciones de seguridad nacional y a veces de otros países u organismos regionales e internacionales.

Esta representación la conforma la historia, la realidad y las vivencias a las que se ha enfrentado la sociedad en términos de presencia de conflictos y las consecuencias que estos han dejado en la sociedad. De igual manera responde al sentido de seguridad nacional y de seguridad individual como ciudadanos o pertenecientes a un determinado grupo ético, político o social, en el cual no se sienten amenazados o, por otro lado, protegidos ante un inminente conflicto armado.
En este caso, se estaría hablando de que se esperan dos tipos de acciones como prerrequisitos para la paz: una acción positiva que radica en la prevención del conflicto y otra negativa la cual se basa en la reacción ofensiva ante una amenaza. Así, las representaciones pueden dilucidar la paz como una forma de protección ante amenazas externas buscando la seguridad, y también, como la respuesta que prevendría determinadas consecuencias como el conflicto.

Para Lederach (2000)el término pax apunta a lo legal como la norma que define las relaciones e interacciones humanas, por lo tanto, es aquella paz dirigida a acciones para mantener el orden social, este orden claro que mantendría la unidad y el orden en la sociedad. Así, una representación social construida con base en este concepto puede girar en torno a la necesidad de la seguridad nacional y la ausencia de conflictos armados como elemento constituyente de la paz.

Otra forma de ver esta representación es la visión de la responsabilidad total que tiene el Estado de brindar seguridad a la ciudadanía por medio de instituciones encargadas de la seguridad nacional (policía, por ejemplo), sin considerar otras medidas que incluyan a la ciudadanía y a la lucha contra la violencia estructural.

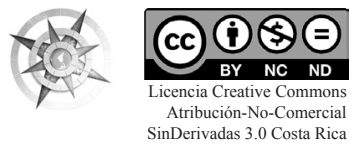




\section{Paz como armonía}

Otra concepción de paz procede de la cultura grecolatina en la cual se entendía como Eirene, concepto que explicaba la ausencia de guerra y la presencia de los Estados de entreguerras. "La Eirene se relaciona con armonía mental, exterior y anímica que se traduce en sentimientos apacibles" (Jiménez, 2009, p. 147). La paz se constituye como sinónimo de armonía, serenidad interior y ausencia de conflictos (Jares, 1999; Lederach, 2000), esto significa que la ausencia de cualquier tipo de conflicto y la tranquilidad interior (del Estado) que esto conlleva son características fundamentales de la paz.

Lo anterior, de acuerdo con Lederach (2000), conlleva a pensar la paz como armonía (Eirene) que describe la unidad interior y social de un grupo, sociedad o Estado, claro que, planteando que la existencia de una tranquilidad interna (estado, sociedad, grupo) influye en la tranquilidad mental individual de la ciudadanía.

Ambas concepciones, la paz negativa y la paz como armonía le han dado a la paz una caracterización de pasividad, es decir, la paz está sujeta a elementos externos, tales como ausencia o no de guerras, conflictos armados o situaciones que limiten la serenidad interior del ser humano. En este sentido, la representación se puede configurar en torno a que la paz depende de circunstancias externas, por lo cual no hay nada que hacer en el ámbito individual, pues esto sería función de las instituciones del Estado (defensa, seguridad). Frente a esto, se puede hablar de elementos de una representación basada en la pasividad ante la construcción o fomento de la paz desde el individuo y la forma en la cual debería asumir su responsabilidad individual en la construcción de una cultura de paz.

Las representaciones de paz como armonía o serenidad interior implican un sentimiento o estado individual que obedece más a situaciones personales que afectan al individuo en su ser interior o a su integridad como ser humano. La pregunta surge, ¿esta serenidad al interior del individuo trasciende más allá de la ausencia o no participación en un conflicto armado o sufrir ante sus consecuencias? Sino más bien, ¿incluye todo aquello que menoscaba la dignidad humana? Y, además, ¿se circunscribe a una visión positiva de condiciones y oportunidades sociales que permiten un desarrollo pleno del individuo? Los sentimientos tranquilos y apacibles pueden ser un resultado de la armonía mental, en este aspecto, la paz como Eirene se concibe como un resultado

120 Referentes conceptuales para el abordaje de las representaciones sociales de la paz Evelyn Cerdas Agüero

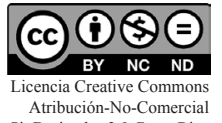


individual, pero reconociendo que los aspectos individuales ejercen influencia sobre la colectividad.

Lo anterior sugiere que las representaciones sociales pueden girar en torno a que la paz está supeditada a la ausencia de conflictos interpersonales como problemas en la comunicación, falta de cooperación, falta de comprensión, agresiones, entre otros. Ante esto, Claggett-Borne (2013) plantea que la paz se suele describir o conceptualizar en razón de lo que no es y no se define en razón de lo que sí es, especialmente se hace énfasis en la ausencia o fin de un determinado acto agresivo o situación peligrosa.

De tal forma que las representaciones basadas en la paz negativa, también remiten a sentimientos, actitudes basadas en un estado mental de no existencia del peligro (en las relaciones, circunstancias, ambiente), por ejemplo, que no haya robos, asaltos, peleas, guerra, invasión, terrorismo, etc.). Esta concepción negativa, también, relaciona Eirene con la ausencia de conflictos interpersonales, puesto que estos podrían interferir en esta armonía interna del individuo, así como cualquier manifestación de la violencia directa que dañe la integridad y menoscabe la armonía individual.
Los alcances entre Eirene y pax, de acuerdo con Lederach (2000) se remiten al hecho de que la pax romana se refería a la ausencia de violencia según lo establecido en la ley "pero no en el sentido de justicia, prosperidad, reciprocidad e igualdad para todos" (p. 18), pero que trasciende hacia la idea de seguridad. Eirene, por otra parte, alude a la armonía basada en la ausencia de conflictos, pese a que el planteamiento parece ser más amplio, se hace necesario preguntar, ¿cuáles serían las situaciones, condiciones, oportunidades que alimentan ese estado de armonía mental del individuo? Además, podría ser que un estado de no acción, de no reconocer la realidad y las necesidades de otras personas genere un estado mental de tranquilidad, sinónimo de pasividad, no acción y despreocupación.

Se puede pensar que si la representación se forma en torno a estos conceptos se enmarca dentro de la visión negativa que no relaciona directamente la paz con los derechos humanos, la igualdad, la justicia, la libertad y la dignidad del ser humano, sino con la ausencia de conflictos interpersonales, grupales y entre Estados. Aunque es importante reconocer que el acceso a oportunidades, la realización de los derechos humanos y la calidad de vida

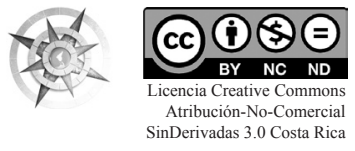

Referentes conceptuales para el abordaje de las representaciones 121

sociales de la paz

Evelyn Cerdas Agüero 
sí generan bienestar y un estado de armonía interna en el ser humano.

\section{La concepción positiva de la paz}

Con respecto a la concepción positiva de la paz, Galtung (2003) plantea que la paz positiva se refiere a la equidad en las relaciones humanas, la ausencia de violencia estructural y cultural. Al mismo tiempo es relacionada con la violencia estructural (Jiménez, 2009; Galtung, 2003; Pascual, 2013), en el sentido de que es necesaria la existencia y lucha por la justicia social y la satisfacción de las necesidades humanas básicas. De acuerdo con Galtung (2003), se entiende en diferentes significados:

- Paz natural: es la cooperación entre especies, la ausencia de luchas, la convivencia con la naturaleza y el cuidado del cuerpo humano. La naturaleza ya no se concibe como un bien utilitario para el ser humano, sino que se trata de que exista un equilibrio ecológico. Las representaciones que se vinculan con esta tipología de paz les dan especial sentido a las relaciones de cooperación entre los seres humanos y con la naturaleza, de forma que no solo somos parte de un ambiente natural, sino que el ser humano coexiste con este.
- Paz positiva directa: se refiere a la bondad hacia uno mismo y hacia otras personas, en el sentido del cuidado del cuerpo, la mente y el espíritu, también, busca la satisfacción de las necesidades básicas humanas (supervivencia, bienestar, libertad e identidad). El amor en este caso es la manifestación de todo lo anterior. Las representaciones que giran en torno a esta concepción se basan en la realización o no de las necesidades humanas básicas como resultado o requisito para que se manifieste la paz, además, pueden plantear la autoestima, aceptación personal, autocuidado y el encuentro con los otros considerando el respeto mutuo y la alteridad ante las necesidades de las otras personas.

- Paz positiva estructural: busca la equidad, la libertad, el diálogo (interior y externo), la integración, la solidaridad y la participación desde las estructuras e instituciones sociales. Se enfoca en el logro de la armonía en tres aspectos del ser humano: el cuerpo, la mente y el espíritu. Sustituye la represión por libertad, la explotación por la equidad, la penetración por el diálogo, la segmentación por la integración, la fragmentación por la

122 Referentes conceptuales para el abordaje de las representaciones sociales de la paz Evelyn Cerdas Agüero

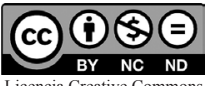

Licencia Creative Commons Atribución-No-Comercial
SinDerivadas 3.0 Costa Rica

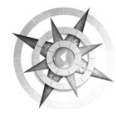


solidaridad y la marginación por la participación (Jiménez, 2011; Galtung, 2003). Se refiere a la ausencia o reducción de la violencia estructural de forma que apuesta por la justicia social. Esta paz también se logra en la estructura individual, en el sentido de paz interior, para lograr la existencia de una armonía entre el cuerpo, el alma y el espíritu.

Las representaciones sociales que permean el concepto de paz positiva estructural se refieren a los siguientes elementos:

1. La búsqueda de la libertad, la justicia, el diálogo, la equidad, la solidaridad, la participación: lo cual se podría manifestar en las opiniones, creencias y actitudes acerca de la necesidad de acciones y cambios para que se generen estos elementos que fundamentan la paz estructural, en este caso se estaría dando una visión negativa que relaciona la paz con la ausencia de estos factores. Por otro lado, pueden girar en torno a propuestas de cómo podría mejorarse la realidad o acercarse más a las actitudes y acciones que fomenten esta forma de paz.

2. La pasividad ante la paz estructural: este planteamiento gira en torno a que esta depende de las instituciones y sistemas sociales, por lo que el individuo no tiene mucha influencia en consolidarla, buscarla, luchar por esta o fomentarla.

3. La paz estructural como un ideal: a esta se pueden circunscribir representaciones que observan la paz como una utopía dadas las diversas condiciones sociales de desigualdad, pobre$z a$, injusticia que no parecen llegar a un punto específico.

4. Una visión integral del ser humano: en esta se considera como una persona que puede alcanzar un desarrollo integral: cuerpo, mente y espíritu.

5. La exposición de manifestaciones de justicia social y las necesidades para que esta se genere: igualdad, acceso a recursos, oportunidades, entre otros.

6. Factores para promover el cambio: el diálogo, la comunicación asertiva, la no discriminación, el respeto a la libertad, la búsqueda de justicia, el trabajo contra estereotipos y las desigualdades sociales.

- Paz positiva cultural: es una paz que justifica y legitima la paz

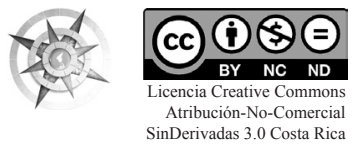

Referentes conceptuales para el abordaje de las representaciones 123

sociales de la paz

Evelyn Cerdas Agüero 
en las diversas esferas como el derecho, el arte, la ideología, la ciencia, la educación y el lenguaje hacia la construcción de una cultura de paz desde diversos espacios como los sistemas educativos y los medios de comunicación. Se refiere a "aspectos de una cultura que sirven para justificar y legitimar la paz directa y la paz estructural" (Galtung, 2003, citado por Jiménez, 2009, p. 154), se refiere a la paz que se va construyendo desde la realidad cultural y que hace frente a la violencia cultural.

La paz cultural se concibe como cultura de paz. La cultura de paz alude a una serie de valores, actitudes, tradiciones, comportamientos y estilos de vida, los cuales se basan en el respeto a la vida, la no violencia, el respeto a principios de soberanía, el respeto y la promoción de los derechos humanos, la solución pacífica de los conflictos, el desarrollo, la protección del medio ambiente, la igualdad, la libertad, la justicia, la democracia, la tolerancia, la solidaridad, la cooperación, la diversidad cultural, en un entorno que favorezca a la paz (Asamblea General de NU, 1999).

\section{Concepciones de la paz y su re- lación con las representaciones sociales}

Las RS en las que se acentúa el concepto de paz positiva cultural hacen énfasis en actitudes, creencias y acciones que justifican la aceptación de las diferencias, la igualdad, el diálogo, el respeto mutuo, la tolerancia y vivencia de los derechos humanos, la resolución pacífica de conflictos, la interculturalidad, el respeto hacia la naturaleza y aquellos aspectos culturales específicos que motivan e incentivan la dignidad del ser humano, esto desde un alcance positivo. En la acepción negativa, se estaría hablando que sería la ausencia de aquellos factores en las diversas esferas (el derecho, el arte, la ideología, la ciencia, la educación y el lenguaje) que no promueven la justicia social, el desarrollo humano, la calidad de vida, el respeto mutuo y factores que no contribuyen a una convivencia pacífica (violencia directa y violencia estructural).

La paz positiva incluye un panorama de justicia social y de desarrollo (Jiménez, 2009) dirigido a la satisfacción de las necesidades básicas del ser humano que le permitirían una mejor calidad de vida. De acuerdo con Claggett-Borne (2013) la paz positiva se relaciona con los

124 Referentes conceptuales para el abordaje de las representaciones sociales de la paz Evelyn Cerdas Agüero 
prerrequisitos que esta debe cumplir, lo cual implicaría que el logro de estos factores mencionados reflejan la paz, hacen referencia a las condiciones que deben darse para el desarrollo del ser humano, la dignidad humana y la convivencia pacífica. Estos prerrequisitos pueden variar desde lo individual hasta lo colectivo, tales como la vigencia de los derechos humanos, la igualdad, la aceptación de las diferencias, la participación democrática, el trabajo cooperativo, la seguridad y el acceso a los recursos.

Así, las representaciones sociales de la paz que se estructuran en el conocimiento del concepto de paz positiva se generan en torno a una aspiración de lo que se desea de la realidad social y de las soluciones sociales, políticas, económicas y culturales que se requieren para que se dé la justicia social, la satisfacción de necesidades y la vida digna del ser humano, lo cual se vincula con el reconocimiento de los derechos humanos de cada persona.

Esta forma de paz se dilucida en las representaciones sociales como un reconocimiento de las necesidades humanas, no para su sobrevivencia, pero sí para su desarrollo y calidad de vida, la búsqueda de acciones para resistir a las situaciones que generan desigualdad, injusticia, pobreza, exclusión, alienación y explotación.

Existen entonces varios aspectos que configuran la representación de paz positiva que se visualizan por medio de las actitudes y acciones que buscan la cooperación, la bondad, la satisfacción de las necesidades básicas, la equidad, la libertad, el diálogo, la participación, la integración, la legitimación de la paz en todas las esferas sociales, culturales, económicas, políticas y legales, de forma que se hace referencia a una visión de paz desde las condiciones para su logro o construcción, los resultados de esta.

\section{Comentarios finales}

De acuerdo con diversos planteamientos, la paz negativa está caracterizada por una concepción de reducción de la violencia, especialmente la violencia directa. Esta tiene su énfasis en evitar los conflictos armados, lo que alude a la seguridad nacional, regional y mundial, sin importar si esa seguridad beneficia a determinados grupos en detrimento de otros. Por otra parte, puede haber ausencia de enfrentamientos bélicos, y no hay paz si hay desigualdad, hambre, desnutrición, injusticia, no acceso al agua, entre otros aspectos. Esta paz se garantiza por un aparato militar que prevenga los conflictos y

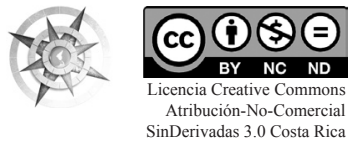

Referentes conceptuales para el abordaje de las representaciones 125

sociales de la paz

Evelyn Cerdas Agüero 
garantice la paz en los períodos entre conflictos armados. Sin embargo, este concepto de paz, de alguna manera, sí acepta la guerra "silenciosa" o con fines pacíficos como los bloqueos comerciales, el aislamiento político y las sanciones económicas.

La paz negativa, si la traducimos a la realidad cotidiana, podría ser descrita como la ausencia de violencia directa (contra el cuerpo, la naturaleza, la propiedad), en tal sentido, es la paz que previene, elimina y trata de reducir las acciones que se cometen contra personas o grupos con efectos directos sobre estas (físicos y psicológicos). Busca hacer frente a la violencia más visible, la violencia directa que tiene una determinada intencionalidad y se ubica en diversos espacios, desde el personal hasta el mundial, pues se genera entre personas, grupos, instituciones, pueblos, etnias, países y regiones.

Por otra parte, la paz positiva busca potenciar la vida del ser humano y de la naturaleza, la igualdad, la justicia y todos aquellos elementos que promueven, garantizan y reconocen la dignidad del ser humano. Además, busca eliminar o reducir las diversas formas de violencia y lograr que exista justicia e igualdad en las sociedades. Esta concepción visualiza la paz como un proceso que es responsabilidad de los individuos y de las instituciones, se relaciona con los cambios culturales, la dignidad humana, el respeto a los derechos humanos, la educación de calidad y el desarrollo sustentable.

Reflexionar sobre elementos conceptuales de la paz negativa y la paz positiva que inciden en las representaciones sociales de la paz permite reconocer que muchos de los significados de paz que surgen de la realidad se construyen por influencia de conceptos que rigen los sistemas sociales, la cultura y las instituciones como por ejemplo las educativas. Sin embargo, no se puede dejar de mencionar la influencia de los medios de comunicación que transmiten determinadas ideologías sustentadas en conceptos y aportes teóricos que tienen un fin específico, así como la historia de un pueblo y las experiencias personales.

Además, permite generar categorías en las cuales se enmarcan las representaciones sociales, lo cual es de gran importancia en la investigación para la paz y posibilita conocer si las representaciones sociales que tiene un determinado grupo están constituidas por conceptos aprendidos, vividos, experiencias o transmisión de conocimientos de representaciones sociales.

126 Referentes conceptuales para el abordaje de las representaciones sociales de la paz Evelyn Cerdas Agüero

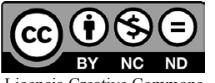

Licencia Creative Commons Atribución-No-Comercial
SinDerivadas 3.0 Costa Rica

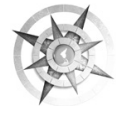


Es importante recalcar que las representaciones sociales están constituidas por creencias, opiniones, actitudes e informaciones, en este trabajo se han escogido las informaciones, al mismo tiempo que se delimita este aspecto a las concepciones. Sin embargo, es necesario mencionar que las informaciones no solo se limitan a los conceptos, sino también a la influencia del contexto, las experiencias, los medios de comunicación, las ideologías y las culturas, entre otros. No es el interés generalizar ni limitar la teoría y los elementos de las representaciones sociales a los conceptos, sino más bien, postular que los conceptos de la paz (positiva y negativa) influyen en la formación de estas y son importantes para la formación de categorías para su análisis.

\section{Referencias}

Abric, J. (2001). Prácticas sociales y representaciones. México: Ediciones Coyoacán.

Araya, S. (2002). Las representaciones sociales. Ejes teóricos para su discusión. Cuaderno de Ciencias Sociales 127, San José, FLACSO. Recuperado de: http://unpan1.un.org/intradoc/groups/public/documents/ICAP/ UNPAN027076.pdf

Asamblea General de Naciones Unidas (1999). Declaración y programa de acción sobre una cultura de paz, A/RES/53/243. Recuperado de:
file://E:/nuevas\%20lecturas $\% 20$ a\%20 marzo2013/paz/declaracion $\% 20$ cult $\% 20$ paz.pdf

Banchs, M.A. (2001). Jugando con las ideas en torno a las representaciones sociales desde Venezuela. Fermentum. Revista Venezolana de Sociología y Antropología, 11(30) 11-32. Recuperado de: http://www.redalyc.org/articulo. oa? id $=70512127003$

Bobbio, N. (1982). El problema de la guerra y las vías de la paz. Barcelona: Gedisa.

Claggett-Borne, E. (2013). Definitions of Peace and Reconciliation in Latin America. International Handbook of Peace and Reconciliation. En: Malley-Morrison, Kathleen, Mercurio, Andrea, Twose, Gabriel (Eds.). Cap. I. Pp. 99-106. New York: Springer Science Business Media.

Cuevas, Y. \& Mireles, O. (2016). Representaciones sociales en la investigación educativa. Estado de la cuestión: producción, referentes y metodología. Perfiles Educativos, XXXVIII (153), 65-83. Recuperado de: http://www.redalyc.org/articulo. oa? id=13246712005

Galtung, J. (2003). Paz por medios pacíficos. Paz y conflicto, desarrollo y civilización. Bilbao: Gernika Gogoratuz.

Ibáñez, T. (2001). Representaciones sociales, teoría y método. En Tomás Ibáñez (Coord.), Psicología social construccionista, pp. 123-216. Guadalajara: Universidad de Guadalajara.

Jares, X. (1999). Educación para la paz. Su teoría y su práctica. Madrid: Editorial Popular.
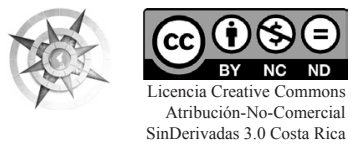

Referentes conceptuales para el abordaje de las representaciones 127 sociales de la paz Evelyn Cerdas Agüero 
Jiménez, F. (2009). Hacia un paradigma pacífico: la paz neutra. Convergencia. Revista de Ciencias Sociales, 16, 141-189. Recuperado de: http://www.redalyc. org/articulo.oa?id=10512244007

Jiménez, F. (2011). Racionalidad pacífica. Una introducción a los estudios para la paz. Madrid: Dikinson.

Jodelet, D. (2007). Imbricaciones entre representaciones sociales e intervención. Representaciones sociales. Teoría e investigación. En: Rodríguez, T. y García, M.L. (Coords.). Pp. 191-1977. Universidad de Guadalajara. Guadalajara: Editorial CUCSH-UDG.

Lederach, J.P. (2000). El abecé de la paz y los conflictos: educar para la paz. Madrid: Los Libros de la Catarata.

Materán, A. (2008). Las representaciones sociales: un referente teórico para la investigación educativa. Geoenseñanza, 13(2), 243-248.
Moscovici, S. (1979). El psicoanálisis, su imagen y su público. Buenos Aires: Editorial Huemul S.A.

Moscovici, S. (2008). Psychoanalysis. Its Image and Its Public. Cambridge: Polity Press.

Pascual, A. (2013). Educación en y para los derechos humanos y la paz: principios y prácticas medulares. Cultura y educación en derechos humanos en América Latina. En Tosi, G., Zenaide, M. De N., Rodino, A.M. y Fernández, M.B. Joao Pessoa - Paraíba, Brasil: Editora Universitária da Universidade Federal da Paraíba (UFPB).

Valencia, S. (2007). Elementos de la construcción, circulación y aplicación de las representaciones sociales. Representaciones sociales. Teoría e investigación. En Rodríguez, T. y García, M.L. (Coords.). Pp. 51-88. Universidad de Guadalajara. Guadalajara: Editorial CUCSH-UDG.

128 Referentes conceptuales para el abordaje de las representaciones 